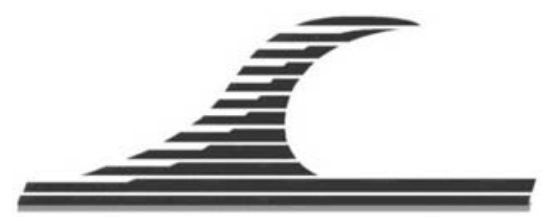

\title{
Un nouveau modèle couplé vagues-courant 3D : développement et validation
}

\author{
Anne-Claire BENNIS ${ }^{1}$, Fabrice ARDHUIN ${ }^{2}$, Tina ODAKA ${ }^{2}$, Franck DUMAS ${ }^{2}$
}

1. UMR CNRS 5805 EPOC, Université Bordeaux 1, Avenue des facultés, 33405 Talence cedex, France. anne-claire.bennis@unicaen.fr

2. Ifremer, BP 70, 29280 Plouzané, France.

ardhuin@ifremer.fr ; tina.odaka@ifremer.fr ,franck.dumas@ifremer.fr

\section{Résumé :}

Un modèle numérique couplant la circulation tridimensionnelle, résolue par le code MARS3D, et l'état de la mer, calculé par le code WAVEWATCH III, est développé pour étudier l'hydrodynamique des zones côtières et littorales. Le principe général du modèle, faisant appel au coupleur PALM est décrit, ainsi que les aménagements apportés aux deux codes existants afin de rendre le couplage possible. Des premiers tests en deux dimensions horizontales ont permis de développer une première version. En trois dimensions, nous montrons que le modèle doit calculer la vitesse quasieulérienne, car il n'existe pas de forme analytique cohérente du forçage par les vagues pour la vitesse lagrangienne. Cet aspect est illustré par un résultat comportant de très fortes erreurs par rapport à la solution analytique, obtenu avec une formulation en vitesse lagrangienne.

Abstract: A numerical model coupling the three-dimensional oceanic circulation, thanks to the MARS3D model, and the sea state computed by the WAVEWATCH III model, is developed in order to study the hydrodynamic of the nearshore zone. The main principle of the coupled model based on the coupler PALM and the necessary modifications made for the coupling procedure to both numerical codes are described. First tests in horizontal two-dimension are made and they allowed us to develop a primary version. For three-dimensional flow, we show that the coupled model must solve the quasi-Eulerian velocity. In fact, a coherent analytical form of the wave forcing for the Lagrangian velocity does not exist. This point is illustrated by one example with large errors in comparison with the analytical solution when solving for the Lagrangian mean flow.

Keywords: Wave-current modeling, Nearshore zone, MARS3D, WAVEWATCH III.

Article issu d'une sélection des XIèmes Journées Nationales Génie Côtier Génie Civil qui se sont tenues aux Sables d'Olonne du 22 au 25 juin 2010.

Accepté le 30 mai 2011, en ligne le 4 novembre 2013.

Pour citer cet article :

BENNIS A.-C., ARDHUIN F., ODAKA T., DUMAS F. (2013). Un nouveau modèle couplé vaguescourant 3D : développement et validation. Revue Paralia, Vol. 6, pp 8.1-8.12. 


\section{Introduction}

L'hydrodynamique littorale est le résultat d'interactions complexes entre états de mer, courants et niveaux d'eau. La compréhension de ces phénomènes hydrodynamiques est par ailleurs nécessaire à la gestion des zones côtières du fait de leur rôle dans le transport sédimentaire, le transport de larves et polluants et le risque qu'ils représentent pour les activités nautiques. Si les phénomènes d'interaction entre l'état de la mer et des courants uniformes sur la verticale sont désormais assez bien connus, plusieurs effets observés semblent être intimement liés à la forte variation du courant sur la verticale, en particulier dans la zone de déferlement, avec le mélange horizontal induit par le cisaillement vertical (SVENDSEN \& PETREVU, 1994), ou la concentration en surface de courants opposés aux vagues (ARDHUIN, 2005), mais aussi sur le plateau continental avec la formation de sous-courants (LENTZ et al., 2008). Afin d'étudier ces phénomènes nous avons donc décidé de développer un outil de modélisation numérique, d'abord à des fins de recherche. Cet outil s'appuie sur deux modèles matures et dont les méthodes numériques, peu diffusives, sont bien adaptées à l'étude de phénomènes présentant de forts gradients. Il s'agit des codes MARS3D (LAZURE \& DUMAS, 2008) pour la circulation océanique, et WAVEWATCH III (ci-dessous WWATCH) pour les états de mer (TOLMAN, 2008 ; ARDHUIN et al., 2009). Afin de minimiser les modifications des deux codes et de permettre un calcul efficace sur un supercalculateur, le coupleur PALM (BUIS et al., 2006) a été utilisé. Dans un premier temps, une version en deux dimensions horizontales a été développée et validée afin de tester notre procédure de couplage. La validation a été faite en zone de déferlement avec des données in situ et sur un cas sans déferlement dont la solution analytique pour l'élévation de surface est donnée par LONGUET-HIGGINS (1967). Un code couplé en trois dimensions a ensuite été développé. La modélisation de la circulation océanique tridimensionnelle en présence de vagues est complexe car à ce jour il n'existe pas encore de modélisation cohérente du flux vertical de quantité de mouvement induit par la propagation des vagues. Si le modèle théorique est basé sur la vitesse lagrangienne, il contient intrinsèquement dans la vitesse résolue la dérive de Stokes et doit donc modéliser correctement ce flux vertical de quantité de mouvement. Si le modèle théorique est basé sur la vitesse quasi-eulérienne, la dérive de Stokes n’est plus contenue intrinsèquement dans la vitesse résolue et donc ce problème épineux de modélisation disparaît. Dans la section 3, nous allons comparer les résultats donnés par le code couplé résolvant la vitesse lagrangienne selon le modèle théorique de MELLOR (2003) avec la vitesse de référence afin de mettre en évidence ce problème de la modélisation du flux vertical de quantité de mouvement induit par la propagation des vagues. 
Un nouveau modèle couplé vagues-courant $3 D$ : développement et validation : 8.3

\section{Principe du couplage et illustration en deux dimensions}

\subsection{Principe du couplage}

Le couplage entre le modèle de circulation MARS3D et le modèle de vagues WWATCH est réalisé à l'aide du coupleur automatique PALM. L'utilisation du coupleur permet une plus grande souplesse dans les échanges entre les deux codes de calcul, ce qui permet une grande liberté dans le choix des variables à transmettre. De plus, l'utilisation de PALM permet une optimisation des modifications à effectuer dans les codes MARS3D et WWATCH, ce qui réduit les sources d'erreur et augmente la lisibilité. Lorsqu'il y a des nouvelles variables à calculer, toutes les opérations sont effectuées dans les programmes intermédiaires wwtomars.f90 et marstoww.f90 (cf. figure 1) dans le but de rentrer dans les codes sources des deux modèles uniquement des variables prêtes à l'emploi. Il s'agit d'un couplage explicite en temps dans lequel les informations sont échangées à chaque pas de temps ou non. Ce couplage explicite n'est pas source d'instabilités pour le modèle couplé. Les grilles de calcul de MARS3D et WWATCH sont pour l'instant les mêmes mais à terme il est prévu qu'elles soient différentes car MARS3D et WWATCH résolvent des processus qui mettent en jeu des échelles spatiales différentes.

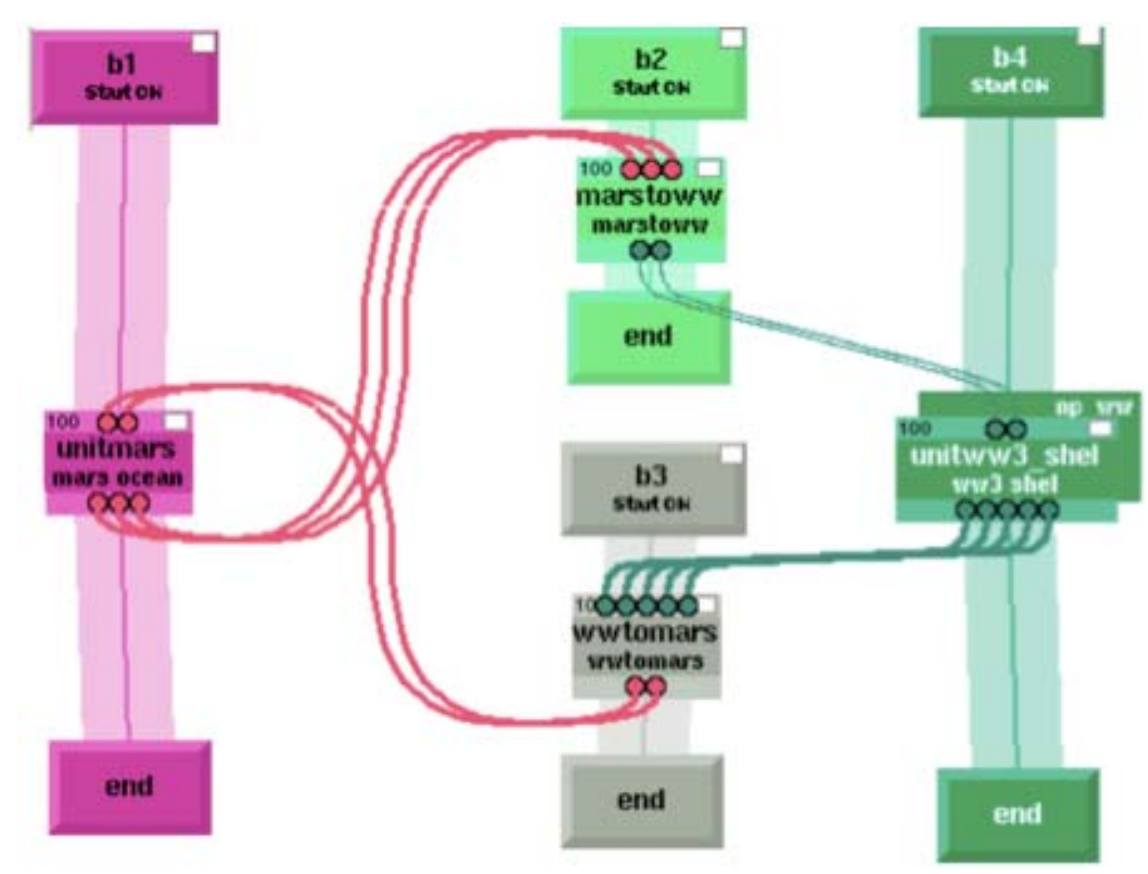

Figure 1. Schéma des interactions entre les différentes composantes du modèle couplé, tel que présenté par l'interface graphique du coupleur PALM. 


\subsection{Modèle couplé en deux dimensions}

\subsubsection{Validation avec les données NSTS}

Le couplage a tout d'abord été testé et validé dans un cas bidimensionnel (2DH). Une approche par tension de radiation (LONGUET-HIGGINS, 1970a ; 1970b) a été utilisée pour forcer le modèle de circulation par le modèle de vagues. Afin d'adapter le modèle de circulation à la région étudiée, les paramétrisations du frottement au fond et de la viscosité horizontale ont été modifiées. Au fond, la paramétrisation de LONGUETHIGGINS (1970a), prenant en compte la modification du frottement au fond par la vitesse orbitale des vagues, a été utilisée. La viscosité horizontale a été paramétrée de telle sorte à considérer une viscosité horizontale qui en zone de déferlement augmente avec l'éloignement à la plage et avec la vitesse de phase des vagues (LONGUETHIGGINS, 1970b). La validation 2DH a été effectuée avec les données de l'expérience NSTS (THORNTON \& GUZA, 1986 ; WU et al., 1985) qui s’est déroulée pendant l'hiver 1980 sur la plage de Leadbetter en Californie. Il s'agit d'étudier la propagation d'une houle oblique avec une incidence de 20 degrés sur une plage plane dont la profondeur décroît régulièrement vers le rivage. L’idée principale est de tester le couplage vagues-courant que nous avons mis en place sur un cas 2DH bien connu dans la littérature. Les auteurs ont donc d'abord cherché à savoir si le forçage était correct puis ils se sont demandés si ce forçage générait le courant mesuré le long de la côte. Un autre indicateur pour le couplage, est le minimum de l'élévation de la surface calculée par le modèle de circulation qui doit correspondre au point de déferlement dont la position est donnée sur le profil de hauteur significative. Ainsi, la comparaison avec les données in situ a été faite sur les profils de hauteur significative et de vitesse le long de la côte en fonction de la distance à la côte. Le code couplé reproduit bien l'intensité du pic de la vitesse le long de la côte par contre la décroissance du courant après le pic est plus faible que celle observée in situ à cause de la paramétrisation de la viscosité horizontale qui est constante en dehors de la zone de déferlement et donc trop forte (figure 2d). La hauteur significative simulée est du même ordre que celle observée in situ même si un léger décalage du point de déferlement est à noter (figure 2c). Le point de déferlement se trouve en $x=111 \mathrm{~m}$, ce qui correspond à la position du minimum de l'élévation de la surface moyenne (figure 2b). Notre procédure de couplage est donc validée sur ce point. Le code couplé reproduit bien les phénomènes de surcote et de décote observés in situ (figure 2b). Sur ce cas test, l'impact de la rétroaction a aussi été étudié et il s'avère que l'on constate des différences très faibles entre le cas forcé et le cas couplé (figures $2 \mathrm{~b}$ et $2 \mathrm{c}$ ). 
Un nouveau modèle couplé vagues-courant 3D : développement et validation : 8.5

a)

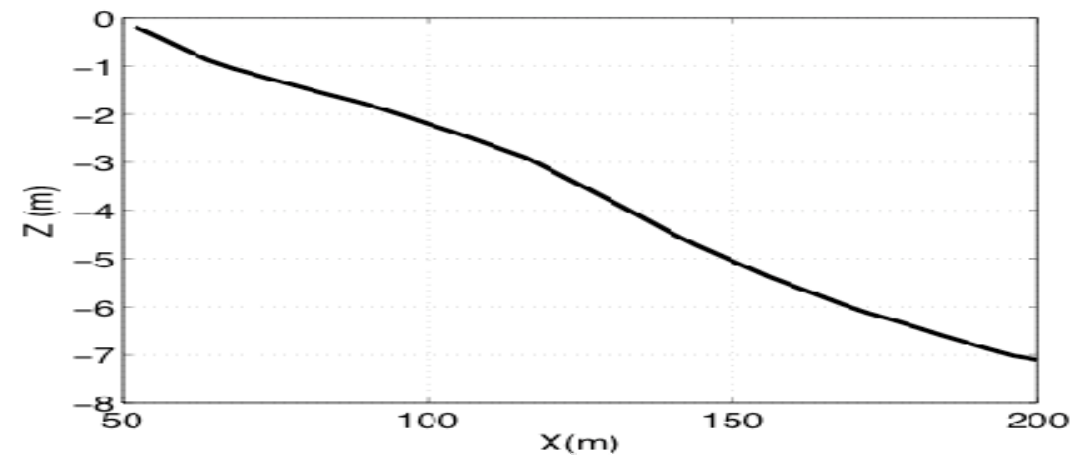

b)

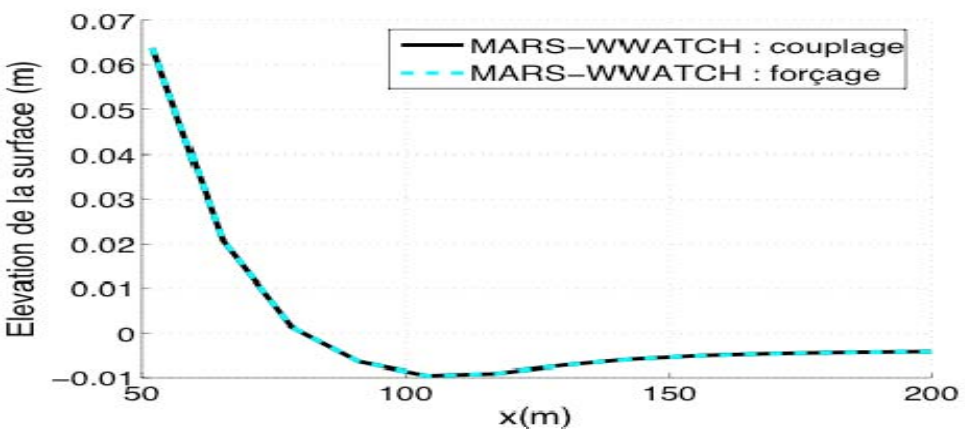

c)

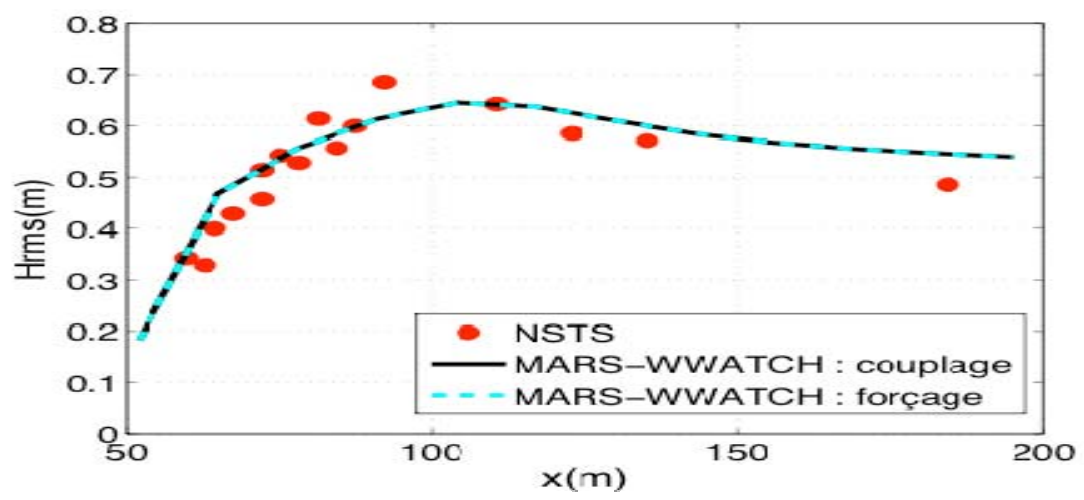

d)

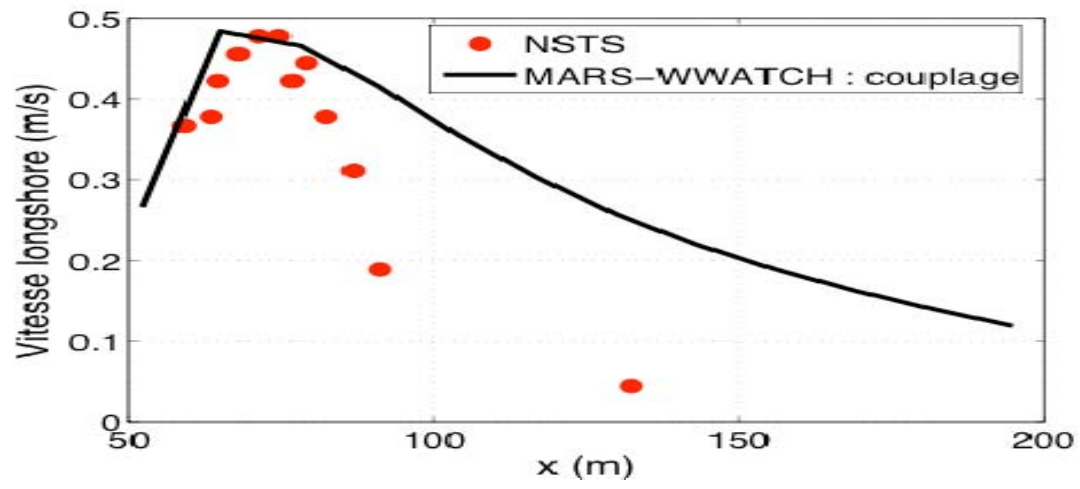

Figure 2. a) Profil bathymétrique. b) Comparaison des profils de l'élévation de la surface moyenne. c) Comparaison des profils de $\mathrm{H}_{r m s}$. d) Comparaison des profils de vitesse le long de la côte (longshore). Sur les figures b), c) et d), les données in situ sont représentées par des points rouges, les données modèles sont tracées en trait plein noir pour le mode couplé et en trait pointillé pour le mode forcé. 


\subsubsection{Validation dans un cas sans déferlement}

Le code couplé en deux dimensions horizontales a aussi servi à étudier un cas sans déferlement. Il s'agit du cas test issu de ARDHUIN et al (2008a) et qui sera utilisé par la suite pour tester les modèles théoriques dans le cas 3D (cf. section 3). La propagation d'une houle d'incidence normale avec une amplitude de $0.12 \mathrm{~m}$ et une période de $5.26 \mathrm{~s}$ sur une bosse dont la profondeur varie de $4 \mathrm{~m}$ à $6 \mathrm{~m}$ est étudiée. Pour ce cas, la solution analytique pour l'élévation de surface est donnée dans LONGUET-HIGGINS (1967) nous permettant de valider la configuration avant de passer au cas 3D. Les deux élévations sont proches (figure 3) sachant que les écarts observés au niveau du plateau proviennent du fait que la solution numérique est calculée avec du frottement au fond alors que ce n'est pas le cas pour la solution analytique.

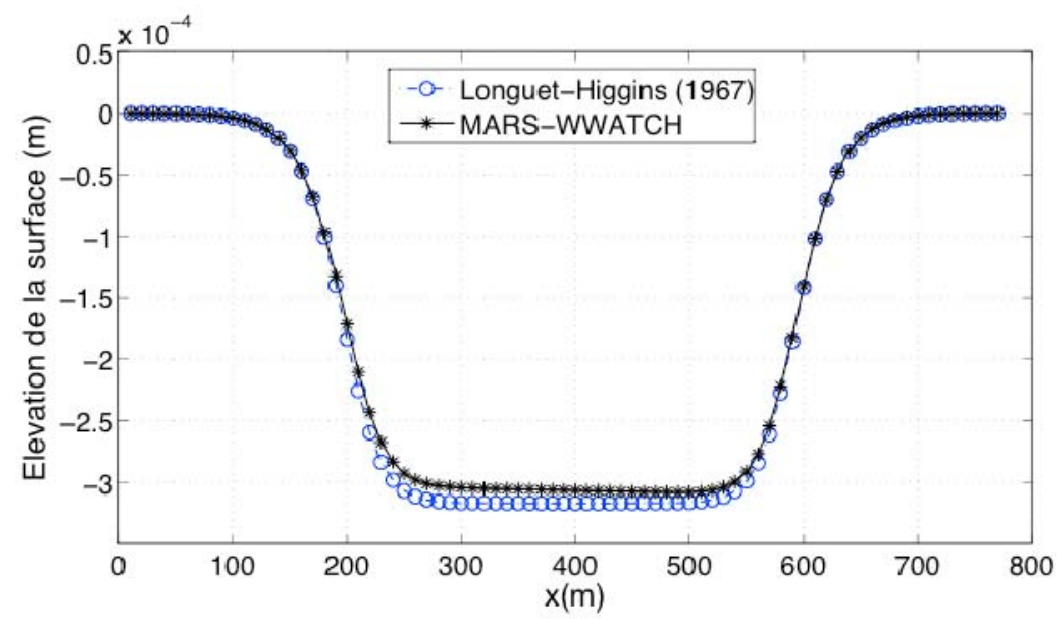

Figure 3. Comparaison de l'élévation de la surface moyenne. Longuet-Higgins (cercles) et modèle en mode forcé (astérisques)

\section{Modèle couplé en trois dimensions}

Dans la section précédente, le modèle théorique utilisé pour prendre en compte l'effet des vagues était celui de PHILLIPS (1977) basé sur les tensions de radiations de LONGUET-HIGGINS (1970a). Ce modèle théorique permet de considérer directement la quantité d'eau déplacée sous l'effet des vagues via la divergence du tenseur des radiations qui force les équations. En trois dimensions, il faut considérer le mouvement vertical des particules et donc modéliser le flux vertical de quantité de mouvement induit par les vagues. La modélisation de ce flux vertical est difficile et donc cela a prolongé jusqu'à peu l'utilisation de modèles en deux dimensions horizontales comme celui de PHILLIPS (1977) ou celui de SMITH (2006). Ainsi le premier problème qui se pose en trois dimensions est le choix des équations de base pour l'évolution de la quantité de mouvement horizontale. En effet, soit on considère l'évolution de la vitesse 
lagrangienne moyenne $U$ qui comprend la dérive de Stokes $U_{s}$ associée aux vagues, soit on considère l'évolution de la vitesse quasi-eulérienne définie à peu près comme $u^{\prime}=U-U_{s}$ (ARDHUIN et al., 2008a). En choisissant de résoudre la vitesse quasieulérienne, le problème difficile de la modélisation du flux vertical de quantité de mouvement induit par les vagues disparaît car $u^{\prime}$ ne contient pas intrinsèquement ce qui vient des vagues. Par contre des termes additionnels apparaissent dans les équations afin de prendre en compte l'effet de la dérive de Stokes $\left(U_{s}, V_{s}, W_{s}\right)$ dans le modèle. Lorsque le modèle théorique concernant la vitesse quasi-eulérienne est intégré sur la verticale, les équations données par SMITH (2006) sont retrouvées. Le jeu d'équations pour la vitesse quasi-eulérienne $\left(u^{\prime}, v^{\prime}, w^{\prime}\right)$ en coordonnée $z$ est le suivant, sachant que celui en coordonnée de terrain est donné dans BENNIS et al. (2011).

$$
\begin{aligned}
& \frac{\partial u^{\prime}}{\partial}+u^{\prime} \frac{\partial u^{\prime}}{\partial x}+v^{\prime} \frac{\partial u^{\prime}}{\partial y}+w^{\prime} \frac{\partial u^{\prime}}{\partial z}-f v^{\prime}+\frac{1}{\rho} \frac{\partial p^{H}}{\partial x}-F_{m, x}^{\prime} \\
& =\left[f+\left(\frac{\partial v^{\prime}}{\partial x}-\frac{\partial u^{\prime}}{\partial y}\right)\right] V_{s}-W_{s} \frac{\partial u^{\prime}}{\partial z}-\frac{\partial J}{\partial x}+F_{d, x}^{\prime}, \\
& \frac{\partial v^{\prime}}{\partial t}+u^{\prime} \frac{\partial v^{\prime}}{\partial x}+v^{\prime} \frac{\partial v^{\prime}}{\partial y}+w^{\prime} \frac{\partial v^{\prime}}{\partial z}+f u^{\prime}+\frac{1}{\rho} \frac{\partial p^{H}}{\partial y}-F_{m, y}^{\prime} \\
& =-\left[f+\left(\frac{\partial v^{\prime}}{\partial x}-\frac{\partial u^{\prime}}{\partial y}\right)\right] U_{s}-W_{s} \frac{\partial v^{\prime}}{\partial z}-\frac{\partial J}{\partial y}+F_{d, y}^{\prime},
\end{aligned}
$$

où $\left(F_{m, x}^{\prime}, F_{m, y}^{\prime}\right)$ et $\left(F_{d, x}^{\prime}, F_{d, y}^{\prime}\right)$ représentent respectivement le mélange et la source de quantité de mouvement venant de la dissipation des vagues. Le terme $J$ représente une pression induite par les vagues. Dans le cas d'ondes linéaires monochromatiques avec une variance de l'élévation de surface $E$, ce terme est indépendant de $z$ et il est défini par $J=(g k E) /[\sinh (2 k D)]$. Les autres notations sont classiques, sauf peut-être $W_{s}$, qui est la composante verticale de la dérive de Stokes, définie précisément par ARDHUIN et al. (2008a). Par ailleurs les équations (1) et (2) doivent être combinées à la conservation de la masse (3) et les conditions aux limites adéquates au fond et en surface (4) doivent être appliquées :

$\frac{\partial u^{\prime}}{\partial x}+\frac{\partial v^{\prime}}{\partial y}+\frac{\partial w^{\prime}}{\partial z}=0$ partout

et $\frac{\partial \eta^{\prime}}{\partial}+\left(u^{\prime}+U_{s}\right) \frac{\partial \eta^{\prime}}{\partial x}+\left(v^{\prime}+V_{s}\right) \frac{\partial \eta^{\prime}}{\partial y}=w^{\prime}+W_{s}$ en surface ,

avec $\eta^{\prime}$ l'élévation moyenne de la surface.

La particularité des équations (1) et (2) par rapport à ce que résout classiquement un modèle de circulation océanique, est la différence entre les vitesses d'advection des 
traceurs qui reste $U$, et la vitesse d'advection de la quantité de mouvement horizontale $u^{\prime}$.

Une autre possibilité, en théorie, est la résolution de l'équation d'évolution de la vitesse lagrangienne U. En transformant la coordonnée verticale $z$ tel que l'on ait : $z=s(x, \zeta, t)=\eta^{\prime}+\zeta D+s^{*}$ avec $\zeta$ variant de -1 à 0 , ceci permettant de prendre en compte la perturbation de la position verticale des particules induite par les vagues, le modèle suivant est obtenu (MELLOR, 2003 ; ARDHUIN et al., 2008b), (avec la profondeur moyenne $\left.D=h+\eta^{\prime}\right)$ :

$$
\frac{\partial U}{\partial t}+U \frac{\partial U}{\partial x}+\frac{\Omega}{D} \frac{\partial U}{\partial \zeta}=\frac{F}{D}
$$

où $\Omega$ est la vitesse verticale en coordonnée $\zeta$ et $F / D$ est l'accélération résultant la somme des forces :

- gradient de pression hydrostatique ;

- divergence des flux de quantité de mouvements horizontaux et verticaux associés aux vagues (tensions de radiation);

- mélange.

Les expressions complètes de $F$ sont données dans les deux articles précités. Nous noterons seulement ici que la divergence verticale des tensions de radiation verticales doit être estimée au même ordre que la divergence horizontale. Or, dans le cas d'un fond en pente, cela demande une solution du mouvement orbital induit par les vagues au premier ordre en pente du fond, et il n'y a pas de solution analytique de ce type. La mise en oeuvre de ce type de modèle demande donc une modélisation complexe des vagues, hors de portée pour les applications pratiques (ARDHUIN et al., 2008b). MELLOR (2003) n'a pas prêté attention à ce problème de cohérence dans l'estimation des différents termes, et il a utilisé la théorie d'Airy, pour des vagues linéaires sur fond plat, pour estimer tous les termes de forçage. Cette approche incohérente du forçage aboutit à des erreurs importantes, comme nous allons le voir. Ainsi, nous avons étudié un cas simple de la propagation de vagues sur une bosse lisse dans la direction $\mathrm{x}$ et invariante dans la direction y (cf. figures 4 et 5), en absence de frottement au fond et de dissipation. La houle se propage sur la bathymétrie avec une incidence normale et la rétroaction des courants sur les vagues n'est pas prise en compte. La solution stationnaire de (1) donne une vitesse lagrangienne de l'ordre de $2 \mathrm{~cm} / \mathrm{s}$ pour une profondeur variant de 4 à $6 \mathrm{~m}$, un période de 5,6 s et une amplitude des vagues de $36 \mathrm{~cm}$ correspondant à une hauteur significative de $1.02 \mathrm{~m}$.

Par contre la résolution numérique de (5) avec notre modèle en mode forcé utilisant l'expression du forçage par les vagues, donnée par MELLOR (2003), aboutit à des vitesses beaucoup plus fortes, de l'ordre de $20 \mathrm{~cm} / \mathrm{s}$, et une solution faiblement nonstationnaire qui, localement (pour $x=200 \mathrm{~m}$ et $x=600 \mathrm{~m}$ ) a presque convergé au bout de 15 minutes de temps simulé. Toutefois, l'advection des régions de fortes vitesses finit 
Un nouveau modèle couplé vagues-courant $3 D$ : développement et validation : 8.9

par entraîner des instabilités numériques lorsque la région de vitesse positive s'étend et rejoint, au milieu du domaine de calcul en $x=380 \mathrm{~m}$, la région de vitesse négative.

Par ailleurs, lorsque le fond est modifié en l'étirant dans la direction $\mathrm{x}$ d'un facteur $1 / \alpha$, les vitesses obtenues pour une profondeur fixe au temps $t=t_{0} / \alpha^{2}$ ne dépendent pas de la pente maximale du fond car l'équation (5) est en similitude de Froude puisque $F(x, t)$ n'est fonction que du champ de vagues à $x$ et $t$. En d'autres termes, les très fortes erreurs de vitesse subsistent sur un plateau continental en pente très douce. Seul le mélange vertical et la tension sur le fond peuvent faire varier la vitesse. Dans le cas présent un mélange vertical assez fort (viscosité turbulente $K_{\mathrm{z}}$ constante égale à 2,8 $\times 10^{-3} \mathrm{~m}^{2} \mathrm{~s}^{-1}$ ) a été testé et permet de réduire la vitesse en surface aux environs $12 \mathrm{~cm} / \mathrm{s}$ (cf. Tableau 1).

Tableau 1. Vitesse lagrangienne à la surface (U) en $x=200 \mathrm{~m}$ pour différentes simulations. Plusieurs types de forçage ont été testés (hauteur significative : $H_{s}=1.02 \mathrm{~m}$ et $H_{s}=0.34 \mathrm{~m}$, période pic : $T_{p}=5.6 \mathrm{~s}$ et $\left.T_{p}=13 \mathrm{~s}\right)$ pour des cas avec et sans mélange vertical (respectivement $K_{z}=2.8 \times 10^{-3} \mathrm{~m}^{2} \mathrm{~s}^{-1}$ et $K_{\mathrm{z}}=0 \mathrm{~m}^{2} \mathrm{~s}^{-1}$ ).

\begin{tabular}{llll}
\hline$H_{s}(m)$ & $T_{p}(s)$ & $K_{z}\left(m^{2} s^{-1}\right)$ & $U\left(m^{-1}\right)$ \\
\hline 1.02 & 5.6 & 0 & 0.1698 \\
0.34 & 5.6 & 0 & 0.0537 \\
0.34 & 13 & 0 & 0.0110 \\
1.02 & 5.6 & $2.8 .10^{-3}$ & 0.1094 \\
0.34 & 5.6 & $2.8 .10^{-3}$ & 0.0185 \\
0.34 & 13 & $2.8 .10^{-3}$ & 0.0026 \\
\hline
\end{tabular}

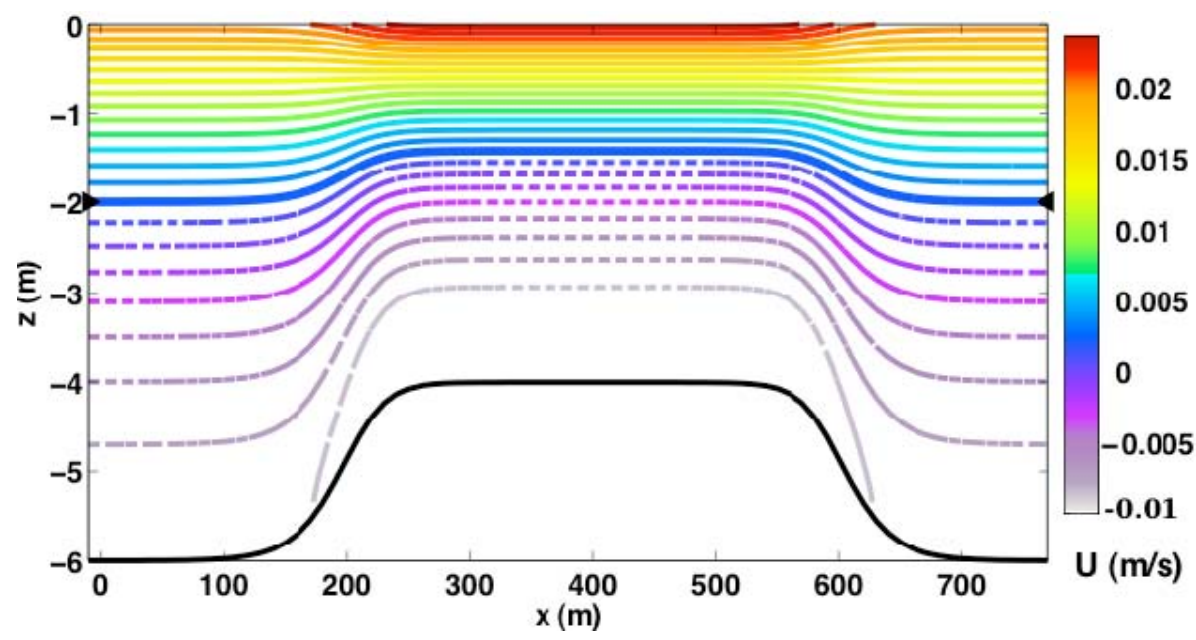

Figure 4. Cas de vagues d'amplitude $0.36 \mathrm{~m}$ (ie. $H_{\mathrm{s}}=1.02 \mathrm{~m}$ ) et de période $5.6 \mathrm{~s}$ sur un fond en pente douce - Vitesse lagrangienne horizontale stationnaire obtenue en ajoutant la dérive de Stokes et la vitesse quasi-eulérienne, solution du $2^{\text {nd }}$ ordre de l'équation de Laplace. Valeurs négatives (trait pointillé) et valeurs positives (trait plein) séparées par le contour zéro (en gras et marqueurs à gauche et à droite). Contours régulièrement espacés entre $-0.01 \mathrm{~m} / \mathrm{s}$ et $0.025 \mathrm{~m} / \mathrm{s}$. 


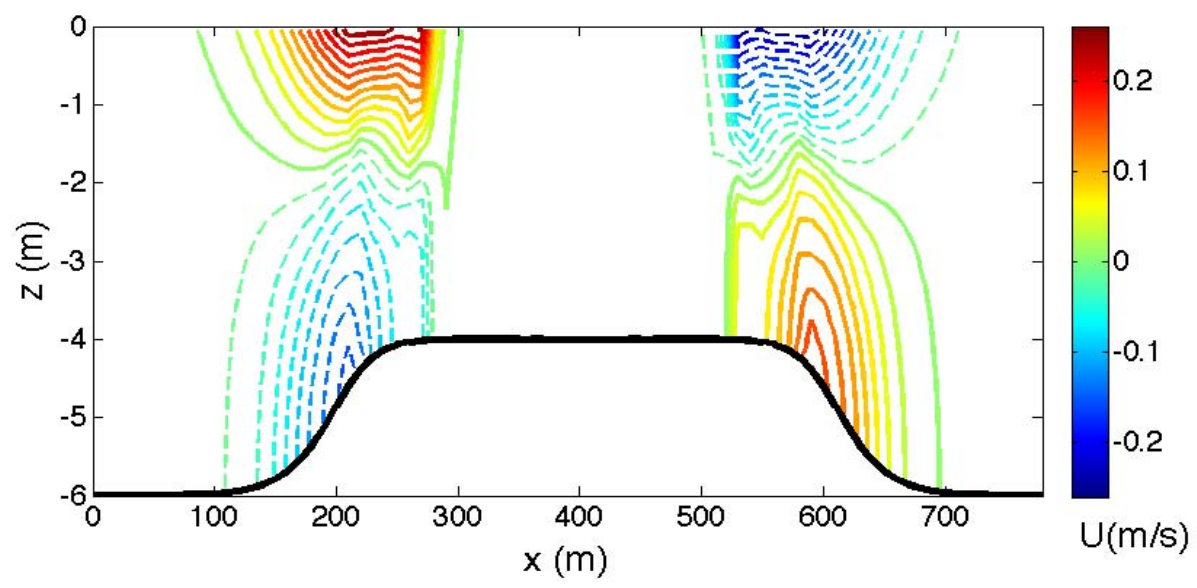

Figure 5. Cas de vagues d'amplitude $0.36 \mathrm{~m}$ (i.e. $\mathrm{Hs}=1.02 \mathrm{~m}$ ) et de période $5.6 \mathrm{~s}$ sur un fond en pente douce - Vitesse lagrangienne horizontale après 15 minutes d'intégration obtenue avec le modèle couplé résolvant les équations erronées de MELLOR (2003).

Valeurs négatives (trait pointillé) et valeurs positives (trait plein). Contours régulièrement espacés entre $-0.25 \mathrm{~m} / \mathrm{s}$ et $0.25 \mathrm{~m} / \mathrm{s}$.

\section{Perspectives}

L’implémentation des équations (1) et (2) dans le code couplé MARS3D-WWATCH III est en cours de réalisation afin de pouvoir à terme s'intéresser à des problèmes tridimensionnels réalistes.

\section{Remerciements :}

A.-C. Bennis remercie l'INSU pour son financement post-doctoral obtenu dans le cadre de l'ANR EPIGRAM (financement $n^{\circ}$ ANR-BLAN-08-0330-01). F. Ardhuin est financé par le Conseil Européen de la Recherche (projet IOWAGA, n²40009) et l’U.S. Office of Naval Research (financement $n^{\circ}$ N00014-10-1-0383).

\section{Références}

ARDHUIN F. (2005). Etat de la mer et dynamique de l'océan superficial. Habilitation à diriger des recherches, Université de Bretagne Occidentale, 318 p.

ARDHUIN F., RASCLE N., BELIBASSAKIS K.A. (2008a). Explicit wave-averaged primitive equations using a generalized lagrangian mean. Ocean Modelling, Vol. 20, pp 235-264. doi:10.1016/j.ocemod.2007.07.001 
Un nouveau modèle couplé vagues-courant $3 D$ : développement et validation : 8.11

ARDHUIN F., JENKINS A.D., BELIBASSAKIS K. (2008b). Commentary on 'the three-dimensional current and surface wave equations' by George Mellor. J. Phys. Oceanogr., Vol. 38, pp 1340-1349. doi:10.1175/2007JPO3670.1

ARDHUIN F., MARIE L., RASCLE N., FORGET P., ROLAND A. (2009). Observation and estimation of Lagrangian, Stokes and Eulerian currents induced by wind and waves at the sea surface. J. Phys. Oceanogr., Vol. 39, pp 2820-2838. doi:10.1175/2009JPO4169.1

BENNIS A.-C., ARDHUIN F., DUMAS F. (2011). On the coupling of wave and threedimensional circulation models: Choice of theoretical framework, practical implementation and adiabatic tests. Ocean Modeling, Volume 40 (3-4), pp 260-272. doi:10.1016/j.ocemod.2011.09.003

BUIS S., PIACENTINI A., DECLAT D. (2006). PALM : A computational framework for assembling high performance computing applications. Concurrency and Computation: Practice and Experience, Vol. 18, pp 231-245. doi:10.1002/cpe.914

LAZURE P., DUMAS F. (2008). An external-internal mode coupling for $3 d$ hydrodynamical model for applications at regional scale (MARS). Adv. Water Resources, Vol. 31, pp 233-250. doi:10.1016/j.advwatres.2007.06.010

LENTZ S.J., HOWD M.F., FREDERICKS J., HATHAWAY K. (2008). Observations and a model of undertow over the inner continental shelf. J. Phys. Oceanogr., Vol. 38, pp 2341-2357. doi:10.1175/2008JPO3986.1

LONGUET-HIGGINS M.S. (1967). On the wave-induced difference in mean sea level between the two sides of a submerged breakwater. J. Mar. Res., Vol. 25, pp 148-153.

LONGUET-HIGGINS M.S. (1970a). Longshore currents generated by obliquely incident sea waves, 1. J. Geophys. Res., Vol. 75, pp 6778-6789. doi:10.1029/JC075i033p06778 LONGUET-HIGGINS M.S. (1970b). Longshore currents generated by obliquely incident sea waves, 2. J. Geophys. Res., Vol. 75, pp 6790-6801. doi:10.1029/JC075i033p06790 MELLOR G.L. (2003). The three-dimensional current and surface wave equations. J. Phys. Oceanogr., 33, pp 1978-1989. doi:10.1175/1520-0485(2003)033<1978:TTCASW>2.0.CO;2 PHILLIPS O.M. (1977). The dynamics of the upper ocean. Cambridge University Press, London, $336 \mathrm{p}$.

SMITH J.A. (2006). Wave-current interactions in finite-depth. J. Phys.Oceanogr., Vol. 36, pp 1403-1419. doi:10.1175/JPO2911.1

SVENDSEN I.A., PETREVU U. (1994). Nearshore mixing and dispersion. Proc. Roy. Soc. Lond. A, Vol. 445, pp 561-576. doi:10.1098/rspa.1994.0078

THORNTON E.B., GUZA R.T. (1986). Surf zone longshore currents and random waves: Field Data and Models. J. Phys. Oceanogr., Vol. 16, pp 1165-1178. doi:10.1175/1520-0485(1986)016<1165:SZLCAR>2.0.CO;2 
8.12 : Revue Paralia - Vol. 6 (2013)

TOLMAN H.L. (2008). A mosaic approach to wind wave modelling. Ocean Modelling, Vol. 25, pp 35-47. doi:10.1016/j.ocemod.2008.06.005

WU C.S., THORNTON E.B., GUZA R. (1985). Waves and longshore currents: Comparison of a numerical model with field data. J. Geophys. Res., Vol. 90, pp 4951-4958. doi:10.1029/JC090iC03p04951 